\title{
Neural Computations for Sound Pattern Recognition: Evidence for Summation of an Array of Frequency Filters in an Echolocating Bat
}

\author{
Roald C. Roverud \\ Department of Biology, University of California, Riverside, California 92521
}

Microchiropteran bats use an auditory sonar system for orlentation and prey capture. Many bats use highly structured constant-frequency (CF) and frequency-modulated (FM) sonar orientation signals. Mechanisms for sound pattern recognition are important for the perception of these and other types of auditory signals. The processing and recognition of FM sound components appears to be important for certain complex perceptual tasks, including target distance perception. I have conducted behavioral studies using artificial echoes to simulate the conditions of a bat flying toward a target. An innate vocalization response of the bat to the simulated approaching target was used to assess the ability of the bat to analyze the structure of and extract distance information from different types of synthetic FM sound patterns. The bat's performance depended on the structure of the artificial echo. The pattern recognition performance of the bats was similar when they were presented with either a naturally structured artificial CF/FM echo or an artificial CF/FM echo containing an FM component consisting of a series of pure tone steps. The ability of the bats to recognize appropriately the structure of an FM signal constructed from a sequence of pure tones depended on the number of pure tone steps in the series. Noctilio was able to recognize FM sound patterns containing 99 or greater pure tone steps. The minimum required number of pure tone steps could be distributed over different frequency ranges. The bats were able to resolve individual tone steps in the series that were separated by at least $100 \mathrm{~Hz}$. These results provide behavioral evidence suggesting the existence of an array of neurons, each of which responds to a specific frequency or narrow range of frequencies in the FM sweep. A critical computation step seems to involve neural summation of information from this array of frequency filters.

[Key words: pattern recognition, neural summation, audition, echolocation, auditory information processing, neural integration, bat]

Pattern recognition is a critical function of mechanisms for sensory information processing. For example, mechanisms for sound pattern recognition are important for the perception of auditory communication signals. In some animals, certain forms of orientation behavior also depend on the processing and recognition of acoustic signals. Microchiropteran bats use an auditory sonar

Received July 8, 1992; revised Oct. 12, 1992; accepted Nov. 16, 1992.

This work was supported by intramural grants from the University of California at Riverside to R.C.R.

Correspondence should be addressed to Roald C. Roverud at the above address.

Copyright (C) 1993 Society for Neuroscience $0270-6474 / 93 / 132306-07 \$ 05.00 / 0$ system known as echolocation for detecting, locating, and recognizing objects in the environment (Griffin, 1958). Bats perceive target features by neural processing of an emitted echolocation call and a corresponding returning echo. Many bats produce highly structured echolocation calls that consist of constant-frequency (CF) and frequency-modulated (FM) elements. The processing and recognition of FM components appears to be important for certain complex perceptual tasks, including target distance perception (Roverud, 1987; Simmons and Grinnell, 1988). Although mechanisms for sound pattern recognition are not well understood, it has been demonstrated in the visual system that neural convergence and summation are important for pattern recognition (Hubel and Wiesel, 1962), and the general occurrence of synaptic summation has been well documented at the cellular level. Certain characteristics of the auditory system in bats suggest that a similar type of integration process may be important for FM pattern recognition. Each primary sensory neuron of the auditory system is tuned to a specific frequency, each of which could potentially serve as an element of a more complex sound pattern. The total array of acoustic neurons from the periphery are tonotopically organized and cover a range of frequencies that are biologically relevant for each species. Certain neurophysiological studies suggest that specific frequencies or frequency segments are processed from an FM sweep. Pollak et al. (1977) have described neurons in the inferior colliculus of Mexican free-tailed bats that show phasic, constant-latency discharge patterns, and have suggested that these neurons are particularly suited for target ranging. Each of these neurons responded to specific frequencies within an FM signal, different neurons responded to different frequencies, and the entire set of these neurons was tonotopically organized (Bodenhamer and Pollak, 1981). In Eptesicus, the functional organization of certain neurons in the nucleus of the lateral lemniscus suggests that only selected frequencies or bands of frequencies of an FM signal are processed by distinct sheets of cells and that different sheets of cells process different select frequencies from the FM sweep (Covey and Casseday, 1986). The behavioral relevance of this type of information or how it may be used during the processing of FM signals is unclear. In this article, I present behavioral evidence supporting the hypothesis that complex sound pattern recognition in bats is accomplished by an analysis process involving neural summation of an array of frequency-tuned neurons, each of which responds to a specific frequency element of the complex FM signal.

\section{Materials and Methods}

A bat searching for a target emits its echolocation calls at a rate of about 5-10/sec (Griffin, 1958). After the bat detects a target and comes to within about 2-3 $\mathrm{m}$ of the target, the bat enters the so-called approach phase of target-directed flight, during which time the echolocation behavior of the bat changes in a stereotypical manner, most strikingly 
characterized by an increase in the repetition rate of the echolocation calls (Griffin, 1958). This stereotyped behavioral response to an approaching target depends on the processing of distance information from the bat's echolocation sounds, which in turn is dependent on the processing and recognition of FM signals (Roverud, 1987; Simmons and Grinnell, 1988).

I have conducted behavioral studies using artificial echoes that simulated the conditions of a target approaching a bat to determine mechanisms for FM sound pattern recognition. Five adult male bats of the species Noctilio albiventris were used in the study. The bats were maintained on a 12:12 hr day/night cycle, and the experiments were conducted at the beginning of the night cycle each day. The bats were trained to hang from a small perch at one end of a dimly lit $7.5 \times 4.5 \times 2.5$ $\mathrm{m}$ room. A speaker and microphone assemblage was located $10 \mathrm{~cm}$ in front of and $45^{\circ}$ below the bat's head. Training procedures or reinforcement was not associated with the presentation of stimuli during the experimental trials. Instead, an innate vocalization response of the bat to the stimuli was used to assess the bat's pattern recognition performance. After the bat was placed on the perch, its vocalizations were recorded by the microphone and monitored on an oscilloscope. Spontaneous vocalizations initiated an experimental trial that consisted of the playback of a predetermined sequence of artificial echoes simulating an approaching target. The artificial echoes were generated with a computer-controlled synthesizer (Wavetek model 650), Hewlett-Packard model 355 attenuators, Krohn-Hite model 7500 amplifier, and a Polaroid electrostatic speaker. The frequency response of the system was flat to within $\pm 3 \mathrm{~dB}$ over the range of presented frequencies. The bat's calls were recorded with a QMC model SM1 microphone and passed through an SA Instrumentation model SA-13T amplitude discriminator, which generated a TTL trigger pulse at the onset of the bat's call. This trigger pulse was fed into a computer-controlled arbitrary waveform generator (Wavetek model 75), which produced a second TTL trigger at a controlled interval from the first. Bats measure the time interval between the emitted call and returning echo to determine target distance (Simmons, 1973). Each trial consisted of the playback of a predetermined sequence of call to echo time intervals. Each of the bat's emitted calls triggered the playback of an artificial echo; the first echolocation call triggered the playback of an artificial echo at $5 \mathrm{~m}$, and each successive call triggered the playback of an artificial echo at a progressively closer predetermined virtual target distance until, at the end of the sequence, the target finally reached a simulated distance of $0.3 \mathrm{~m}$, at which time the echo playback was terminated. The change in distance over successive bat calls was adjusted to maintain a rate of virtual target movement of about $5 \mathrm{~m} / \mathrm{sec}$, which is within the range of the normal flight speed of the bats (Vaughan, 1970).

The bat's behavioral response to the simulated approaching target was determined in the following manner. The calls emitted by the bat during the presentation of an approaching target were recorded by a microphone, digitized by an analog-to-digital converter (R.C. Electronics ISC-16), and stored on a computer. The time interval between successive vocalizations was determined by subsequent analysis. Each trial was scored as showing echolocation behavior similar to bats searching for prey or approaching prey under natural conditions. Search phase behavior was identified by the bats emitting pairs of pulses consisting of a CF signal followed about $40 \mathrm{msec}$ later by a CF/FM call at a rate of about 6-10 pairs/sec. Thus, the two-call sets were separated by a relatively long interval of about $125 \mathrm{msec}$. The bats occasionally emitted a three-call set in place of the much more typical two-call set. A trial was scored as showing approach stage behavior when all of the following criteria were observed: (1) at the beginning of the trial the bat emitted calls in the typical search pattern, (2) during the trial the bat dropped the $C F$ signal and began to emit a sequence of six or greater $C F / F M$ calls at a rate of at least 30 calls/sec, and (3) the bat returned to the typical search vocalization pattern following the termination of the approaching artificial target. Thus, a designation of approach behavior required the bats to emit a sequence of six or greater CF/FM calls where the interval between the onset of each successive call over the entire sequence was about $33 \mathrm{msec}$ or less. Each bat was presented with 20 trials of a simulated approaching target each day, and the percentage of the trials where they responded with the stereotypical approach stage behavior was determined. The temporal and spectral structure of the artificial echoes was systcmatically modificd over successive days, and the effect on the percentage of approach stage responses exhibited by the bat was recorded.

Frequency spectra were generated by recording the artificial echo signals with a Bruel and Kjaer 1/4-inch microphone, digitizing the sig- nals with an analog-to-digital converter (R.C. Electronics ISC-16), and using digital signal processing software (Interactive Laboratory System) to implement a fast Fourier transform analysis of a two-tone step segment of the signal.

\section{Results}

Response of the bat to a virtual approaching target

When hanging from the perch, the bats spontaneously emitted pairs of pulses consisting of a CF signal (about $8 \mathrm{msec}, 75 \mathrm{kHz}$ ) followed about $40 \mathrm{msec}$ later by a CF/FM call (CF about $4 \mathrm{msec}$, $75 \mathrm{kHz}$; FM about $4 \mathrm{msec}, 75-55 \mathrm{kHz}$ ) at a rate of about $6-10$ pairs/sec. This echolocation behavior was similar to bats searching for prey under natural conditions (Roverud and Grinnell, 1985a). When the bats were presented with a simulated approaching target based on an artificial CF/FM echo that matched the bat's own call, they dropped the CF signal and began emitting a sequence of CF/FM calls (CF about 2-4 msec, $75 \mathrm{kHz}$; FM about $4 \mathrm{msec}, 75-35 \mathrm{kHz}$ ) at an increased rate of about 30 calls/ sec. This echolocation behavior was similar to bats approaching prey under natural conditions (Roverud and Grinnell, 1985a). The bats were presented with this naturally structured signal at the beginning and end of each experiment, and there was little variation in the performance of the bats from one presentation to the next in a given bat during the course of the experiments.

The bat's performance depended on the structure of the artificial echo. When presented with an artificial CF/FM signal that was similar in structure to the bat's own echolocation sound, the bats responded with approach stage behavior $69 \%$ of the time (see Fig. 2). This signal consisted of a 4 msec CF component at $75 \mathrm{kHz}$ followed by a $4 \mathrm{msec} F M$ component sweeping continuously from 75 to $55 \mathrm{kHz}$. This and all subsequent signals had a $0.2 \mathrm{msec}$ rise/fall time and were presented at $75 \mathrm{~dB}$ SPL at the bat's listening position. Systematic modifications in the frequency pattern over time of the FM component of the artificial CF/FM echo resulted in changes in the percentage of the trials to which the bats responded with approach stage behavior.

\section{Effects of the number of pure tone steps}

The initial experiment involved the presentation of artificial CF/FM echoes consisting of a $4 \mathrm{msec}$ CF component at $75 \mathrm{kHz}$ followed by a 4 msec FM component composed of a series of discrete pure tone frequency steps, digitally switching from step to step in a seamless manner from 75 to $55 \mathrm{kHz}$. When the signal switched from one tone step to the next, the new sine waveform began at the precise phase and amplitude where the sine waveform of the previous step had ended. For example, one step may end and the next step begin at the positive amplitude peak of the sine wave. The maintenance of amplitude and phase continuity while executing an ultrafast (<500 nsec) switch between any two frequencies prevented switching transients and spectral distortions at the junctions between the steps. Therefore, the energy of the signal was confined to the frequencies of the pure tone steps (Fig. 1).

The ability of the bat to recognize an FM signal and respond with approach stage behavior depended on the number of pure tone frequency elements in the FM signal (Fig. 2). When the FM component consisted of 125 or 99 pure tone elements, the bats responded with approach stage behavior at a performance level that was not significantly different from that seen when the bats were presented with a continuously sweeping FM signal ( $p>0.05$, paired comparison $t$ test; Fig. 2). When presented with an FM component consisting of 90 or fewer pure tone steps, the extent to which the bats responded with approach 

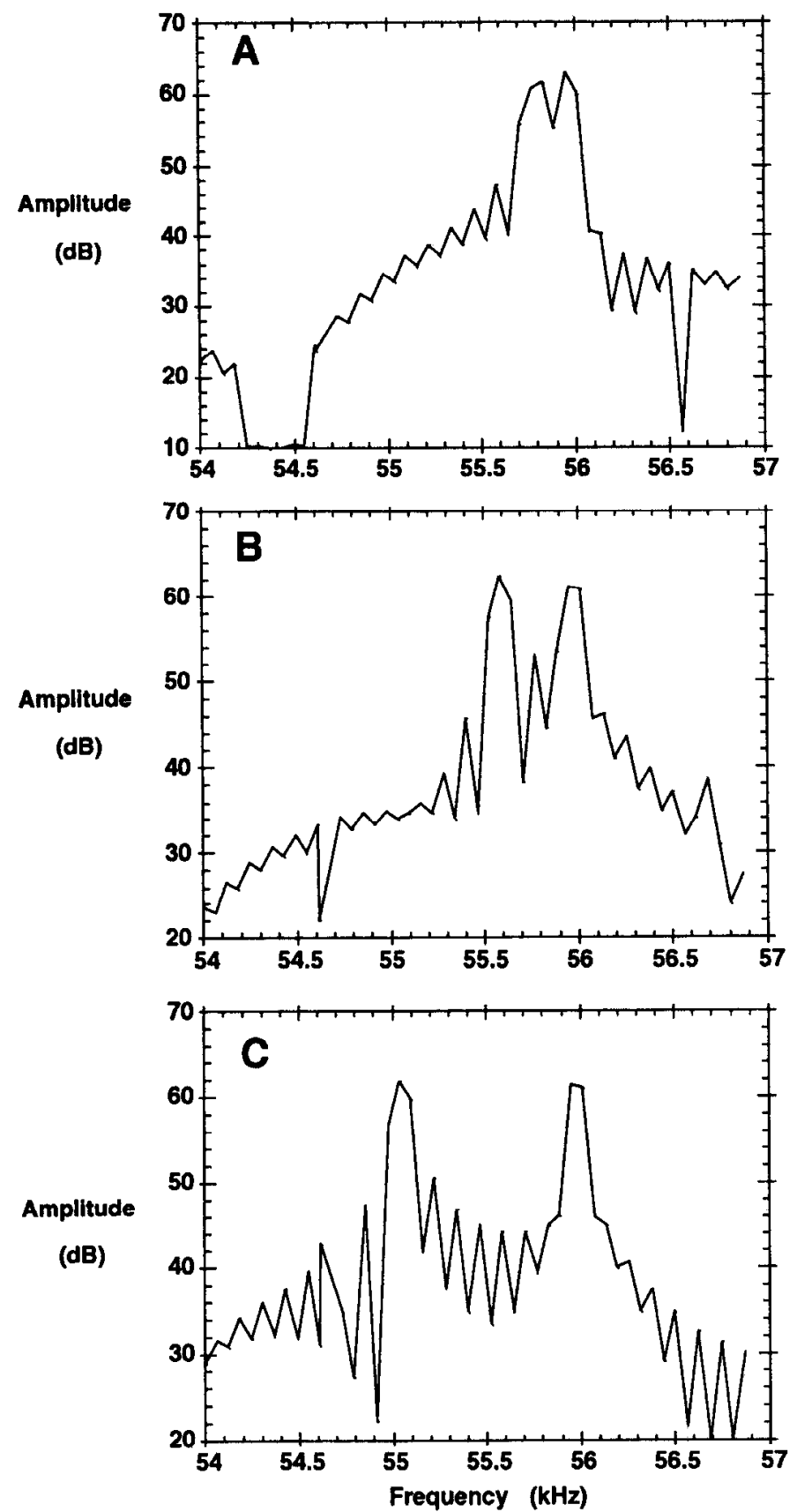

Figure 1. Frequency spectra generated by fast Fourier transform analysis of a two-tone step segment of artificial FM signals composed of a sequence of pure tone steps. The difference in frequency between the two pure tone steps was $200 \mathrm{~Hz}(A), 400 \mathrm{~Hz}(B)$, and $1000 \mathrm{~Hz}(C)$.

stage behavior sharply declined to values significantly below that seen with a continuous FM sweep ( $p<0.05$; Fig. 2). The results suggest that there is a fairly sharp threshold for the number of pure tone frequency elements required for FM pattern recognition and the induction of approach stage behavior, since there was a sharp rise in the percentage of approach stage behavior between 80 pure tone frequency steps and 99 steps.

\section{Effects of the frequency difference between steps and step duration}

In the above experiment the FM signals always sweep from about 75 to $55 \mathrm{kHz}$ in $4 \mathrm{msec}$. Therefore, as the number of pure

\section{$\%$ Approach Phase Responses}

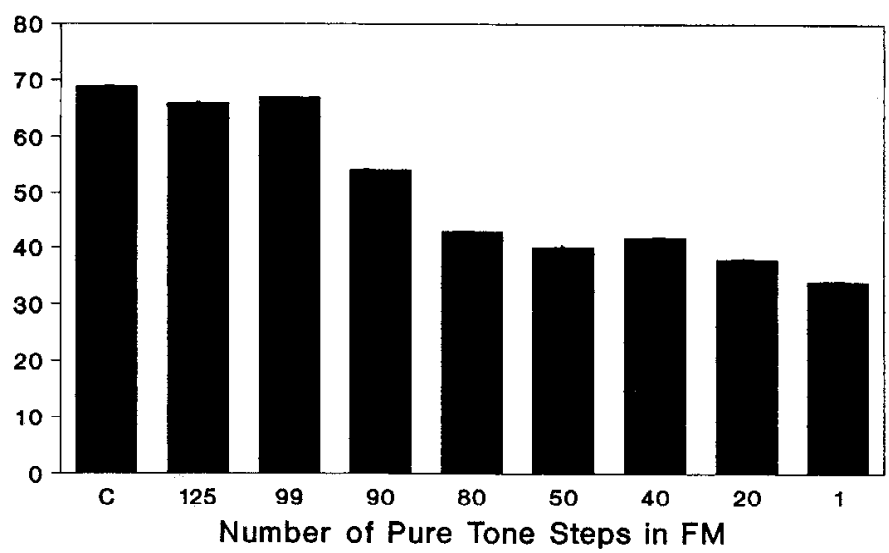

Figure 2. The degree to which the bats responded with approach stage echolocation behavior when presented with a simulated approaching target. The artificial CF/FM echoes contained FM components composed of various numbers of pure tone frequency steps. $C$, continuous FM sweep. The SDs from the mean values indicated for each step number were, for $C, 10.8 ; 125,6.5 ; 99,11.5 ; 90,9.6 ; 80,9.6 ; 50,9.4$; $40,10.4 ; 20,11.9 ; 1,13.5 . n=100 / \mathrm{bar}$.

tone frequency steps in the FM component was reduced, there was a corresponding increase in frequency change between steps and in the duration of each step. To determine if the bat's ability to recognize FM signals depended on the modifications in these parameters rather than simply on the number of individual frequency steps, initially the frequency difference between steps and then individual step duration was independently varied. When step number and step duration were held at their original values of 99 and $0.04 \mathrm{msec}$, respectively, but the frequency difference between steps was changed to $100 \mathrm{~Hz}$ or to $400 \mathrm{~Hz}$, the performance of the bats was not significantly different from that seen with the original frequency difference of $200 \mathrm{~Hz}(p>$ 0.05 ; Fig. 3). When step number and the frequency difference between steps were held at their original values of 99 and 200 $\mathrm{Hz}$, respectively, but step duration was modified to $0.02 \mathrm{msec}$ or to $0.08 \mathrm{msec}$, the performance of the bats was not significantly different from that seen with the original step duration of 0.04 msec ( $p>0.05$; Fig. 4). Therefore, the decline in percentage of approach stage behavior was not due to modifications in the frequency difference between steps or individual step duration. A combination of an increase in both frequency difference between steps and step duration was also not the critical factor, since when the bats were presented with an FM signal with 99 steps of $0.08 \mathrm{msec}$ duration separated by $400 \mathrm{~Hz}$, their performance level was not significantly different from that observed with the original signal consisting of 99 steps of $0.04 \mathrm{msec}$ duration separated by $200 \mathrm{~Hz}(63 \%$ approach stage responses, $p>0.05)$.

\section{Effects of the frequency range of the FM sweep}

To determine if the overall frequency range of the FM signal was the limiting factor rather than the number of individual elements within the FM sweep, the bats were presented with FM signals containing either 99 or 80 pure tone steps that were distributcd over different frequency ranges. When step number and individual step duration were kept constant at 99 and 0.04 msec, respectively, and the frequency difference between steps was set at 100,200 , and $400 \mathrm{~Hz}$, the individual tone steps were 


\section{\% Approach Phase Responses}

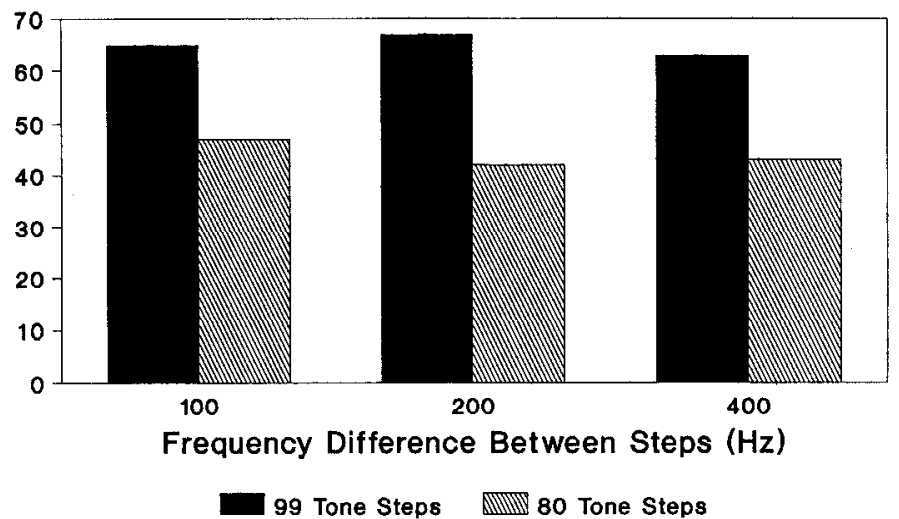

Figure 3. The degree to which the bats responded with approach stage echolocation behavior when presented with a simulated approaching target. The artificial CF/FM echoes contained FM components composed of either 99 or 80 pure tone frequency steps. The frequency difference between the individual steps was varied. The SDs from the mean values indicated were, for $100 \mathrm{~Hz} / 99$ steps, $10.0 ; 100 \mathrm{~Hz} / 80 \mathrm{steps}$, 7.6; $200 \mathrm{~Hz} / 99$ steps, $11.5 ; 200 \mathrm{~Hz} / 80$ steps, $5.7 ; 400 \mathrm{~Hz} / 99$ steps, 7.6; $400 \mathrm{~Hz} / 80$ steps, 7.6. $n=100 /$ bar.

evenly distributed over about 10,20 , and $40 \mathrm{kHz}$, respectively. In all cases the FM component swept down from the CF frequency of $75 \mathrm{kHz}$. As previously mentioned, the bats responded to all three of these signals in a manner similar to that of a continuous FM signal sweeping over $20 \mathrm{kHz}$ (from 75 to 55 $\mathrm{kHz}$; Fig. 3). For all three FM frequency ranges, when the number of steps was reduced to 80 the bats' performances sharply declined to values significantly below those observed with 99 steps ( $p<0.05$; Fig. 3). Therefore, it appears that an FM sweep must contain approximately 99 or greater pure tone frequency elements, but these individual elements can be distributed over various total frequency ranges.

\section{Effects of the duration of the FM sweep}

To determine if the overall duration of the signal was the restrictive parameter instead of the number of individual elements within the FM sweep, the bats were presented with FM components containing either 99 or 80 pure tone steps extending over different signal durations. With a step number of 99 and frequency difference between steps set at $200 \mathrm{~Hz}$, individual step durations of $0.02,0.04$, and $0.08 \mathrm{msec}$ resulted in FM sweeps of about 2,4 , and $8 \mathrm{msec}$, respectively. When the bats were presented with these signals, they responded in a manner similar to that seen with a continuous FM sweep of $4 \mathrm{msec}$ duration (Fig. 4). For all three signal durations, the bats' performances again sharply declined to values significantly below those observed with 99 steps when step number was reduced to 80 ( $p<0.05$; Fig. 4). Thus, FM sweeps of various overall durations must contain about 99 or greater individual frequencies.

\section{Resolution of the frequency difference between tone steps}

To determine the minimum frequency difference between tone steps that the bats can resolve, the frequency difference between the steps was systematically reduced while step number and step duration remained constant at 99 steps and $0.04 \mathrm{msec}$, respectively. When the frequency difference between each evenly distributed pure tone step was set at either $100 \mathrm{~Hz}, 200 \mathrm{~Hz}$, or 400

\section{\% Approach Phase Responses}

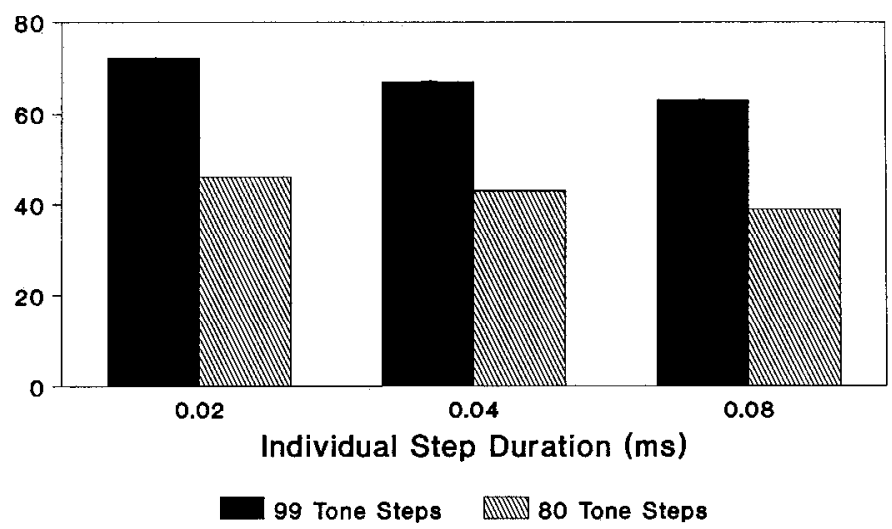

Figure 4. The degree to which the bats responded with approach stage echolocation behavior when presented with a simulated approaching target. The artificial CF/FM echoes contained FM components composed of either 99 or 80 pure tone frequency steps. The duration of each individual step was varied. The SDs from the mean values indicated were, for $0.02 \mathrm{msec} / 99 \mathrm{steps}, 9.1 ; 0.02 \mathrm{msec} / 80 \mathrm{steps}, 6.5 ; 0.04$ $\mathrm{msec} / 99$ steps, $11.5 ; 0.04 \mathrm{msec} / 80$ steps, $9.1 ; 0.08 \mathrm{msec} / 99$ steps, 6.7 ; $0.08 \mathrm{msec} / 80$ steps, 8.9 . $n=100 /$ bar.

$\mathrm{Hz}$, the performances of the bats were not significantly different from that seen with the standard continuous FM sweep $(p>$ 0.05 ; Fig. 5). When the frequency difference between the steps was reduced to $50 \mathrm{~Hz}$, the performance of the bats decreased to a level significantly below that seen with a continuous FM sweep $(p<0.05)$. When the frequency difference between the evenly distributed tone steps was reduced, the entire extent of the FM sweep proportionately decreased. To determine if the change in overall frequency range of the FM signal was responsible for the decrease in the bat's performance rather than the change in frequency difference between neighboring steps, oddnumbered steps were maintained at a difference of $400 \mathrm{IIz}$ (FM from about 75 to $55 \mathrm{kHz}$ ) while even-numbered steps were varied in position between their higher and lower neighboring

\section{\% Approach Phase Responses}

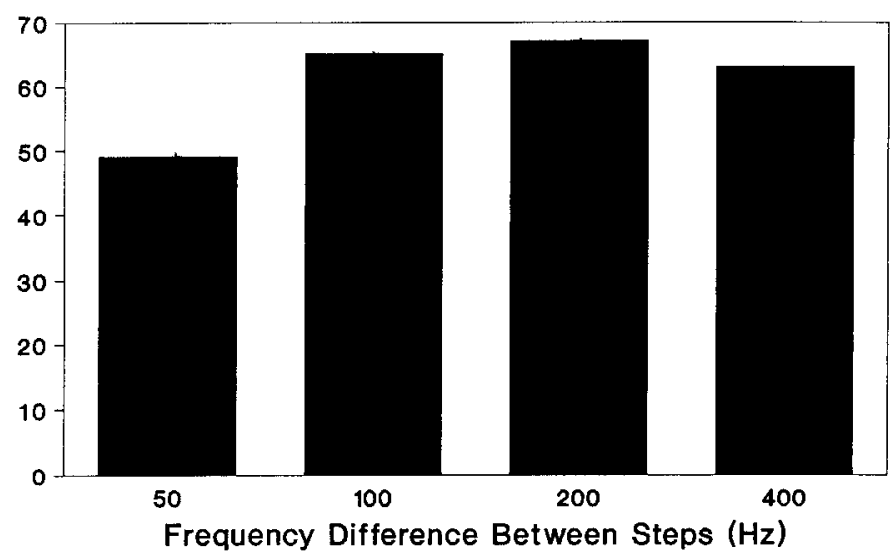

Figure 5. The degree to which the bats responded with approach stage echolocation behavior when presented with a simulated approaching target. The artificial CF/FM echoes contained FM components composed of 99 pure tone frequency steps. The frequency difference between the evenly distributed steps was systematically varied. The SDs from the mean values indicated were, for $50 \mathrm{~Hz}, 9.8 ; 100 \mathrm{~Hz}, 10.0 ; 200 \mathrm{~Hz}$, $11.5 ; 400 \mathrm{~Hz}, 7.6 . n=100 /$ bar. 


\section{\% Approach Phase Responses}

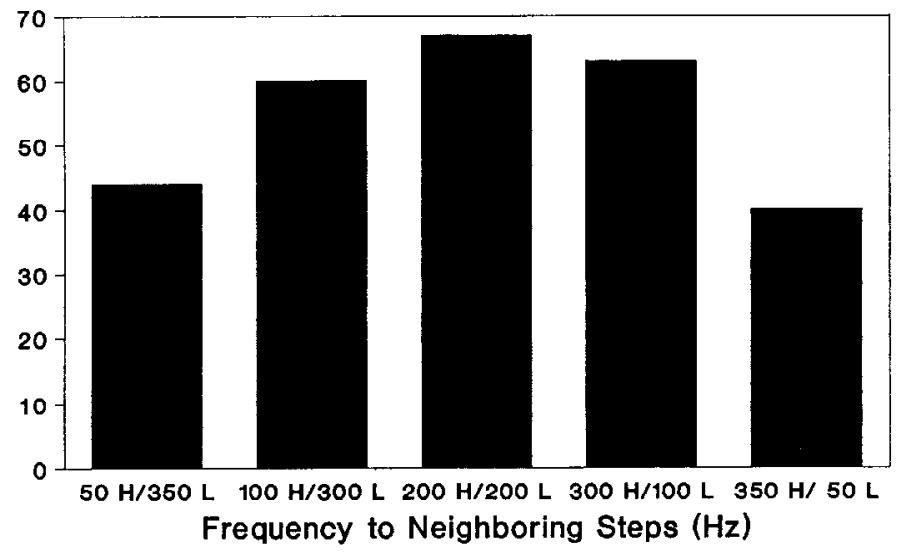

Figure 6. The degree to which the bats responded with approach stage echolocation behavior when presented with a simulated approaching target. The artificial CF/FM echoes contained FM components composed of 99 pure tone frequency steps. The frequency difference between evenly distributed odd-numbered steps was maintained at $400 \mathrm{~Hz}$. The frequency of all even-numbered steps was varied between those of the neighboring higher $(H)$ and lower $(L)$ odd-numbered steps. The SDs from the mean values indicated were, for $50 \mathrm{H} / 350 \mathrm{~L}, 8.3 ; 100 \mathrm{H} / 300$ $\mathrm{L}, 10.3 ; 200 \mathrm{H} / 200 \mathrm{~L}, 11.5 ; 300 \mathrm{H} / 100 \mathrm{~L}, 9.4 ; 350 \mathrm{H} / 50 \mathrm{~L}, 10.2 . n=$ $100 / \mathrm{bar}$.

steps (Fig. 6). When the even-numbered steps were separated by at least $100 \mathrm{~Hz}$ from both the higher or lower neighboring steps, the bats' performance was not significantly different from that observed with a continuous FM sweep $(p>0.05)$. When the even-numbered steps were placed $50 \mathrm{~Hz}$ from either the higher or lower neighboring frequency steps, the bats' performance decreased to a level significantly below that seen with a continuous FM sweep $(p<0.05)$. Thus, the bats can resolve individual tone steps within a series of tone steps constituting an FM sweep that are separated by about $100 \mathrm{~Hz}$ or more.

\section{Discussion}

The observed approach phase behavioral response of the bat to a virtual approaching target clearly depends on the extraction of distance information from the artificial echoes. Under natural conditions bats exhibit approach phase echolocation behavior only when they are flying toward a target that is less than about 2-3 $\mathrm{m}$ away (Schnitzler and Henson, 1980). Approach phase behavior is also observed in a stationary bat if an object is moved toward the bat (R. Roverud, personal observation). Moreover, the only stimulus feature that was altered in the virtual approaching target simulator was the time interval between the bat's emitted call and the returning artificial echo. Bats measure the time interval between the emitted call and returning echo to determine target distance (Simmons, 1973). The various functional tasks of echolocation, such as distance perception, depend on information processing steps for recognizing particular sound structures. Target distance perception is dependent on the processing and recognition of FM sound patterns (Roverud, 1987; Simmons and Grinnell, 1988). Thus, assessment of approach phase behavior is a reliable indicator of the ability of the bat to process and recognize FM signals.

The results of this study demonstrate that the FM component of the bat's CF/FM echolocation sound can be represented by a series of pure tone steps. The number of tone steps in the series is a critical determinant of the bat's ability to recognize an FM signal. It appears that Noctilio requires a minimum of about 99 pure tone steps in order to classify a sound pattern as a biologically relevant FM signal. This value is an approximation since the results reveal that the bats require more than 90 steps and that 99 steps are sufficient. Therefore, it is possible that the minimum number of steps that the bats actually require lies somewhere between 91 and 99 steps. In any event, 99 steps are clearly sufficient, and therefore this value is designated as the known minimum value. These results pertain to FM structures used for distance perception and the induction of approach phase responses, although it is conceivable that similar mechanisms are important for analyzing different kinds of information or for sound pattern analysis in other systems.

There appears to be a fairly sharp threshold for the number of pure tone frequency elements required for FM pattern analysis and the induction of approach stage behavior, since there was a sharp decline in the percentage of approach stage behavior between 99 and 80 pure tone steps. Presumably the same type of information can be obtained from processing a naturally structured FM component or an FM signal composed of only 99 pure tone elements. If the number of pure tone elements is moderately reduced to 80 , however, the bats seem to perceive the FM signal no differently than one with as little as 20 tone steps or even a single tone step at the bat's own CF frequency. This suggests that the number of tone steps within an FM sweep must exceed a specific threshold level. FM signals containing numbers of tone steps above or below this threshold value are perceived in significantly different ways. CF/FM signals with FM components containing a number of steps above this threshold value seem to be perceived as naturally structured FM signals, while CF/FM signals with FM components containing less than the threshold number of steps seem to be perceived in a manner similar to signals containing only the CF component. Roverud and Grinnell (1985b) have provided evidence suggesting that the CF component alone is sufficient for target detection while the entire CF/FM signal is needed for accurate distance determination in Noctilio. Presumably, when the bats were presented with a CF signal or a CF/FM signal consisting of an FM component containing up to about 90 steps, they detected the virtual approaching target but, at best, only obtained ambiguous information concerning target distance. Perhaps the combination of clear-cut target detection but ambiguous distance determination causes the bat to respond occasionally with approach phase behavior in an attempt to locate or track the unknown position of the target. In any event, the percentage of approach phase responses to a single pure tone echo at the bat's CF frequency is significantly different from that to a naturally structured $\mathrm{CF} / \mathrm{FM}$ echo, and hence the bats must be obtaining substantially different kinds of information from the two different signals.

In the initial experiment the bats were presented with CF/ FM signals containing FM elements that always swept from about 75 to $55 \mathrm{kHz}$ in $4 \mathrm{msec}$. As the number of pure tone frequency steps in the FM component was reduced, the overall rate of frequency change remained constant, but there was a corresponding increase in frequency difference between each step and in the duration of each step. For example, when the FM signal consisted of 99 pure tone steps there was a frequency difference of $200 \mathrm{~Hz}$ between each step and an individual step duration of $0.04 \mathrm{msec}$. In comparison, an FM signal consisting of 50 steps had a frequency difference of $400 \mathrm{~Hz}$ between each 
step and an individual step duration of 0.08 msec. It appears that the observed changes in the ability of the bat to recognize an FM signal sweeping from 75 to $55 \mathrm{kHz}$ were due to modifications in the number of pure tone steps rather than the changes in the frequency difference between steps and step duration. This was shown by the observation that when tone step number was held constant at 99 and either the frequency difference between steps or step duration was altered, the bat's performance remained at the same level as that observed with a simulated natural FM signal. A combination of an increase in both frequency difference between steps and step duration was also not the critical factor for changes in the bat's performance, since when the bat was presented with a 99 step FM signal where the frequency difference between steps and step duration were both increased to values equal to those of a 50 step signal (which the bats did not recognize), the bats' performance remained at the same high level as that seen with the simulated natural continuous FM signal.

The requirement for 99 separate frequencies is independent of rate of frequency change over time, at least between 2500 and $10,000 \mathrm{kHz} / \mathrm{sec}$. The rate of frequency change depends on both the frequency difference between steps and the duration of each step. In the initial experiment the decrease in step number resulted in a corresponding increase in frequency difference and step duration in such a way that the rate of frequency change remained constant. When the number of steps fell to 90 or below, the occurrence of approach behavior declined sharply even though the overall rate of frequency change over time remained constant. When step number was kept at 99 and either the frequency difference between steps or step duration was independently altered, which varied the rate of frequency change, the bats responded with approach stage behavior at the same relatively high level.

As the bandwidth of a typical continuously sweeping FM signal increases, both the number of frequencies and the total range of frequencies over which the entire signal extends increase. The results of this study suggest that the number of frequencies within an FM sweep that the bat can independently resolve, rather than the total frequency range of the signal, is important for FM pattern recognition. It is significant that the minimum required number of pure tone steps could be distributed over different frequency ranges. For example, an FM sweep consisting of 99 pure tone steps distributed evenly over $10 \mathrm{kHz}$ (FM from about 75 to $65 \mathrm{kHz}$ ) was equivalent in effect to an FM sweep containing 99 pure tone steps distributed evenly over $40 \mathrm{kHz}$ (FM from about 75 to $35 \mathrm{kHz}$ ). Moreover, the bats recognized and processed distance information from an FM sweep containing 99 steps distributed over only $10 \mathrm{kHz}$ (FM from about 75-65 kHz), but they did not recognize an FM sweep containing 80 steps distributed over a much larger total bandwidth of $32 \mathrm{kHz}$ (FM from about $75-43 \mathrm{kHz}$ ). At the very least, the total array of frequency filters appears to be capable of potentially gathering information over the entire range of frequencies of a typical approach phase FM sweep. However, it appears that input from only a certain portion of the total array is sufficient to meet the minimum requirements of the computations for FM recognition and the induction of an approach phase behavioral response. Moreover, it seems that input from various combinations of different elements of the total array of frequency filters can be used to meet the threshold value of the summing process. Perhaps different degrees of input from the total array are required for different perceptual tasks.
It appears that Noctilio can resolve pure tone elements within an FM sweep that are separated by at least $100 \mathrm{~Hz}$. When the required number of evenly distributed pure tone steps within an FM sweep are separated by less than about $100 \mathrm{~Hz}$, the bats do not effectively recognize the signal. Presumably the bats could no longer resolve each pure tone step within the signal and receive information from the necessary number of steps. It appears that the reduction in frequency difference between neighboring frequencies is important for the effect, rather than the decrease in the total extent of the FM sweep, since when the total range of the FM sweep was maintained at a relatively broad $20 \mathrm{kHz}$ bandwidth, the bats no longer recognized the signal when alternate tone steps were placed less than $100 \mathrm{~Hz}$ from either a higher or lower neighboring tone step.

The array of frequency filters hypothesis presented in this report would predict that the minimum bandwidth of a continuously sweeping FM signal that the bats could recognize would depend on the frequency resolution of the individual filters and the minimum number of active filters required to achieve the threshold value of the summing computation. The results demonstrate that Noctilio can resolve pure tone elements within an FM sweep that are separated by at least $100 \mathrm{~Hz}$. This suggests that each individual frequency filter has a frequency resolution of about $100 \mathrm{~Hz}$ and therefore processes information from a distinct $100 \mathrm{~Hz}$ segment of an FM sweep. Moreover, the results show that the bats require the activation of 99 or greater frequency filters. A combined analysis of these two findings would predict that the bats would require a continuous FM sweep with a bandwidth of about $9.9 \mathrm{kHz}$ or greater $(100 \mathrm{~Hz} / \mathrm{step} \times 99$ steps $=9.9 \mathrm{kHz}$ ). A continuous FM sweep of $5 \mathrm{kHz}$, for example, would only stimulate about 50 different frequency filters, which would be inadequate for FM recognition. Psychophysical studies of Noctilio suggest that the bats require a continuous FM sweep with a bandwidth of about $11 \mathrm{kHz}$ or greater in order to discriminate the difference in distance between two simultaneously presented targets (Roverud and Grinnell, 1985b). These results support the array of frequency filters hypothesis since a continuous FM sweep of $11 \mathrm{kHz}$, and a filter resolution of $100 \mathrm{~Hz}$ would provide information from about the minimum number of distinct frequency filters that the present study suggests is required for FM pattern recognition.

The results of this behavioral study suggest a model for part of the neural computations used for FM sound pattern analysis in Noctilio. The study suggests the existence of an array of neurons, each of which responds to a specific frequency or narrow range of frequencies in the FM sweep. The neural computation for FM sound pattern recognition appears to require the integration of information derived from about 99 or greater of these frequency-tuned neurons. The minimum number of frequency elements required for the summing computation, however, can be variously distributed over different total frequency ranges. Presumably, input from different combinations of components of the total array of frequency filters can serve to meet the minimum requirements of the summing computation. This suggests some sort of central integrator that analyzes information from the total array of frequency filters involved in FM pattern recognition. It appears that the integrated activity from the total array must exceed a definite level. The level of activity from the entire array would depend on the number or proportion of individual filters that are active. The mechanism by which the summing computation is made is unclear, although possible mechanisms include the convergence of the total array onto 
feature detector neurons or a more complex population analysis of activity from the critical array of frequency filter neurons.

\section{References}

Bodenhamer RD, Pollak GD (1981) Time and frequency domain processing in the inferior colliculus of echolocating bats. Hearing Res 5:317-335.

Covey E, Casseday JH (1986) Connectional basis for frequency representation in the nuclei of the lateral lemniscus of the bat, Eptesicus fuscus. J Neurosci 6:2926-2940.

Griffin DR (1958) Listening in the dark. New Haven: Yale UP.

Hubel DH, Wiesel TN (1962) Receptive fields, binocular interaction and functional architecture in the cat's visual cortex. J Physiol (Lond) 160:106-154.

Pollak Gd, Marsh DS, Bodenhamer RD, Souther A (1977) Characteristics of phasic on neurons in inferior colliculus of unanesthetized bats with observations relating to mechanisms for echoranging. $J$ Neurophysiol 40:926-942.

Roverud RC (1987) The processing of echolocation sound elements in bats: a behavioural approach. In: Recent advances in the study of bats (Fenton MB, Racey P, Rayner JMV, eds), pp 152-170. Cambridge: Cambridge UP.

Roverud RC, Grinnell AD (1985a) Discrimination performance and echolocation integration requirements for target detection and distance determination in the CF/FM bat, Noctilio albiventris. J Comp Physiol [A] 156:447-456.

Roverud RC, Grinnell AD (1985h) Echolocation sound features processed to provide distance information in the CF/FM bat, Noctilio albiventris: evidence for a gated time window utilizing both $\mathrm{CF}$ and FM components. J Comp Physiol [A] 156:457-469.

Schnitzler H-U, Henson OW (1980) Performance of airborne animal sonar systems. I. Microchiroptera. In: Animal sonar systems (Busnel R-G, Fish JF, eds), pp 109-181. New York: Plenum.

Simmons JA (1973) The resolution of target range by echolocating bats. J Acoust Soc Am 54:157-173.

Simmons JA, Grinnell AD (1988) The performance of echolocation: acoustic images perceived by echolocating bats. In: Animal sonar: processes and performance (Nachtigall PE, Moore PWB, eds), pp 353386. New York: Plenum.

Vaughan TA (1970) Flight patterns and aerodynamics. In: Biology of hats, Vol I (Wimsatt WA, ed), pp 195-216. New York: Academic. 